\title{
Adenoid Cystic Carcinoma of Buccal Mucosa: A Rare Case Report
}

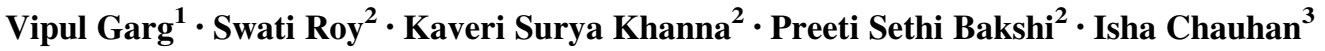

Received: 24 July 2015/Accepted: 7 December 2015/Published online: 11 December 2015

(C) Association of Otolaryngologists of India 2015

\begin{abstract}
Adenoid cystic carcinoma is a malignant neoplasm most commonly originating in salivary glands of head and neck region. Among intra oral adenoid cystic carcinoma, buccal mucosa is among the rarest sites. We report a case of adenoid cystic of buccal mucosa in a 40 -year old female. We have discussed the clinical features, histopathology, diagnosis and treatment along with a brief review of the relevant literature. Although the buccal mucosa is an uncommon site for adenoid cystic carcinoma, the relatively indolent growth pattern of this case and its location which is rather atypical for this type of salivary gland malignancy primarily warrants the necessity behind reporting of this case. Secondly, adenoid cystic carcinoma should be considered in the differential diagnosis of mass of buccal mucosa. It is important to identify such cases rather early and surgical removal with adequate margins is the treatment of choice.
\end{abstract}

Keywords Carcinoma - Adenoid cystic .

Malignant neoplasm $\cdot$ Salivary gland disorders

\section{Introduction}

Salivary gland neoplasms constitutes a diverse group of tumors, exhibiting different histological characteristics and variable clinical behavior patterns $[1,2]$. This diversity in

Swati Roy

dr.swatiroy@gmail.com

1 Department of Oral and Maxillofacial Surgery, Uttaranchal Dental and Medical Research Institute, Dehradun, India

2 Department of Oral and Maxillofacial Pathology, Yamuna Institute of Dental Sciences and Research, Gadholi, Yamunanagar, India

3 Government Dental College and Hospital, Shimla, Himachal pradesh, India the clinical and histological pattern demands for expertise in pathology. A proper diagnosis and treatment is mandatory for salivary gland neoplasms because of their infiltrative behavior and association with late loco-regional recurrence and distant metastasis [3].

Adenoid cystic carcinoma (ACC) is a low-grade malignant tumor arising within the salivary glands that accounts for fewer than $5 \%$ of all head and neck tumors and approximately $10-15 \%$ of salivary gland tumors [3, 4]. Since the first description in 1853, this neoplasm underwent numerous name changes until being given its current name by Spies in 1930. These can arise from all major salivary glands including the parotid, sublingual, submaxillary glands and minor salivary glands. Although, minor salivary gland of the oral cavity is the most common site of this but involvement can occur in almost any site with a secretory gland component and has been reported in sites such as the lacrimal gland, breasts, uterine cervix, esophagus, lungs, and prostate [5]. ACC is known for its prolonged clinical course, perineural invasion, multiple recurrence rates and delayed onset of distant metastasis. Lymph node metastases are unusual; hematogenous spread, often to the lungs is quite characteristic, metastasis to kidney being extremely rare [68]. Here we present a case of adenoid cystic carcinoma of the right buccal mucosa that was surgically excised and healed uneventfully.

\section{Case History}

A 40-year-old female reported with a chief complaint of pain and swelling on the right side of face since 1 year. A year ago, no specific treatment was sought for the same. Extra orally there was facial asymmetry with swelling seen on the right side of the face on the cheek (Fig. 1). Facial 


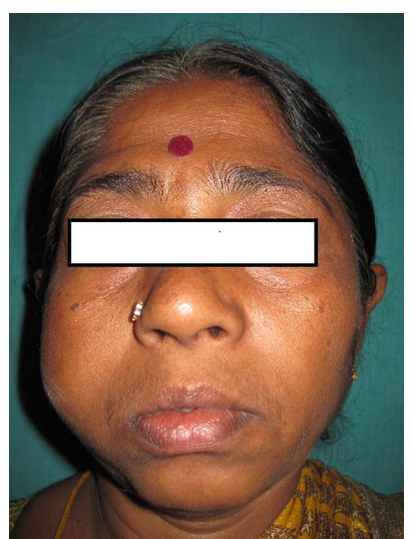

Fig. 1 Extraoral diffuse swelling present on the right cheek

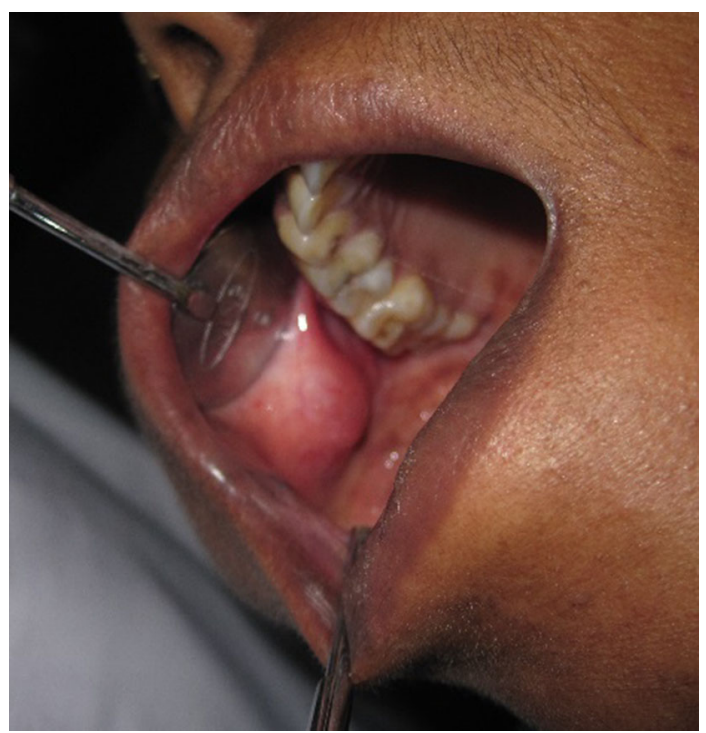

Fig. 2 Intra oral swelling on the right buccal mucosa w.r.t the occlusal line. No surface ulceration evident

nerve function was normal. Intraoral examination revealed a firm lesion of the right buccal mucosa measuring $1.5 \mathrm{~cm} \times 1 \mathrm{~cm}$ in greatest dimension (Fig. 2). Palpation revealed a submucosal firm nodule that was not freely mobile. There was no surface ulceration. The overall clinical picture of the lesion was quite indolent. No lymphadenopathy was detected.

There was mild pain associated with the lesion but no paresthesia was associated with the lesion. Excisional biopsy under local anaesthesia was planned for the patient. After the fibres of buccinators were dissected, demarcation of the lesion was noticed and it was excised in toto. Post operative recovery of the patient was uneventful. The tissue was subjected for histopathological examination. The histopathological examination showed few groups of sheets of uniformly sized darkly staining basaloid cells exhibiting solid growth pattern interspersed by duct like spaces filled with mucinous material with cells showing vesicular nuclei and minimal mitosis (Fig. 3a). Few areas exhibit cribriform and tubular pattern of arrangement of the tumor cells separated by collagenized connective tissue stroma (Fig. 3b). On the basis of histopathological findings, final diagnosis was given as adenoid cystic carcinoma (grade 2).

Post operative necessary clinical and radiological investigations were performed which were within normal limits. The patient is being followed up till date and is still free of disease.

\section{Discussion}

Adenoid cystic carcinoma was first described by Theodor Bilroth in 1856, as cylindromas, in his histological studies where he described it as long amorphous compartments called "cylinders". It is only recently that the tumor has been renamed as adenoid cystic carcinoma (ACC) (Kaiser), which is a rare tumor accounting for $<1 \%$ of all head and neck malignancies and $10 \%$ of all salivary gland neoplasms [8]. ACC is an uncommon form of malignant neoplasm that arises most commonly in the major and minor salivary glands of the head and neck. ACC make up about $6 \%$ of all salivary gland tumors. They make up $15-30 \%$ of submandibular gland tumors, $30 \%$ of minor salivary gland tumors, and $2-15 \%$ of parotid gland tumors [9].

The long natural history of this tumor, its propensity for perineural invasion, and its tendency for local recurrence are well known. Although it presents a widespread age distribution, peak incidence occurs predominantly among women, between the 5th and 6th decades of life. It is a slowly growing but highly invasive cancer with high recurrence rate. Lymphatic spread to local lymph nodes is rare. Hematogenous spread, however, occurs often in the course of the disease. Perineural spread of ACC has long been recognized. The literature revealed that the region of Gasserian ganglion to be the most common site of involvement (35.8\%) [9].

Similar to the literature, the case that we are reporting is also seen in a female and incidence is in the 5th decade of life.

The reported case presented as an indolent swelling of the buccal mucosa that was not found to be infiltrating into the adjacent tissue and was easily separated and excised out. There was no evidence of any local infiltration, lymphatic or perineural spread.

Microscopically, the ACC is composed of a mixture of myoepithelial cells and ductal cells that can have a varied arrangement. Histopathologically, ACC presents three patterns, cribriform, tubular and solid. The cribriform 


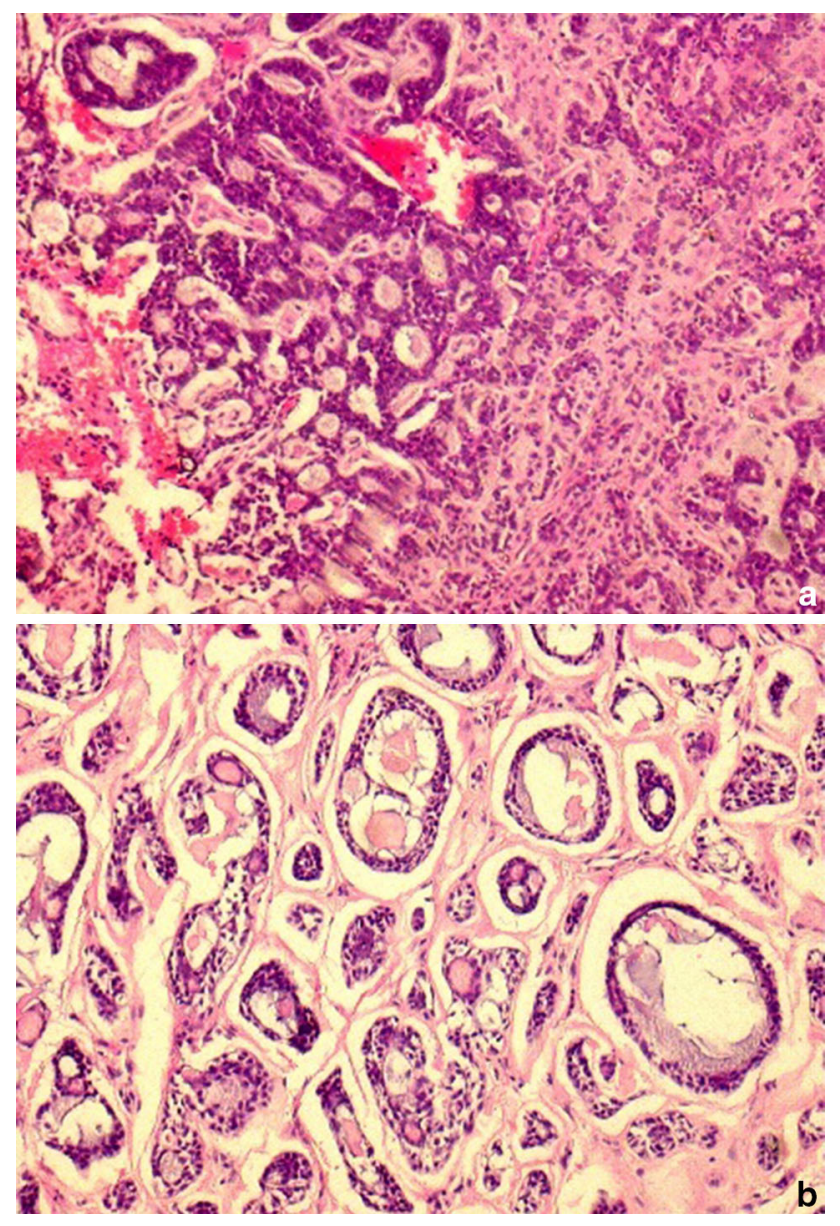

Fig. 3 a Uniformly sized darkly staining cells arranged in variable pattern $(10 \times)$. b Cribriform Pattern: duct like arrangement with mucinous secretory material $(40 \times)$

(glandular) pattern is the most classic and best recognized appearance, characterized by islands of basaloid epithelial cells that contain multiple cylindric, cyst-like spaces resembling Swiss cheese. These spaces often contain a mildly basophilic mucoid material, a hyalinized eosinophilic product, or a combined mucoid hyalinized appearance. The tubular variant is characterized by glandular spaces of elongated tubules lined by epithelial cells and surrounded by single or multiple layers of basaloid cells; the glandular lumens are either empty or contain secretion. The solid variant is composed of solid epithelial islands with central areas of necrosis; the cells are small, basophilic and hyperchromatic densely granulated nucleus. Mitotic figures are generally scarce in cribriform and tubular areas; however they are easily visualized in solid standards that have been associated with the worst prognosis $[10,11]$. The microscopic evaluation of the present case showed both the features of cribriform and solid variant.

Szanto et al. have defined a three tier grading system which takes into account the histomorphologic pattern and percentage of the solid component. This has been regarded as a useful prognostic indicator. Grade 1 ACCs are well differentiated and composed of tubular and cribriform patterns without solid components; grade 2 ACCs are characterized by a pure cribriform pattern or mixed with $<30 \%$ of solid areas; and grade 3 ACCs are tumors with marked predominance of the solid pattern [12]. The present case was falling under the diagnosis of grade 2 ACC. We performed a thorough microscopic evaluation of the specimen for marginal clearance and were found to be free of tumor cells.

The treatment aspect of the tumor is chiefly surgical although in some cases surgery has been successfully coupled with radiotherapy. Radiation therapy as the sole treatment modality seems to be inadequate for ACC. Unlike most other type of salivary gland carcinoma, distant metastasis is far more frequent in ACC (as lungs, liver, kidneys, pelvic girdle) [9]. Lymphatic spread is far less common in this tumor.

In our patient surgical excision as the sole treatment was preferred as the lesion was easily excised out and the margins were found to be clear of tumor cells. Regular follow up of the patient has not revealed any signs of recurrence or metastasis till date. The underlying principles in all ACC therapy are that tumor cells extend well beyond the clinical or radiographic margins and that this tumor undergoes not only perineural invasion but perineural spread. Therefore, it generally requires excision with the widest margins possible. The overall prognosis relates to several factors [8].

\section{Conclusion}

Adenoid cystic carcinomas have a variable prognosis. The 5-year survival rate is $75 \%$, but 10 -year survival rate is only $20 \%$, and survival at 15 years is about $10 \%$ [9]. Other factors which adversely affect the prognosis are perineural spread, distant metastasis and recurrent local lesions. The present case was seen as an indolent localized swelling of the buccal mucosa giving a picture of fibroma, lipoma, neuro fibroma, leiomyoma, oral focal mucinosis, granular cell tumor etc., but the histopathological examination revealed a adenoid cystic carcinoma, so it is important to consider it as a differential diagnosis of localized soft-tissue swelling involving buccal mucosa. The margins of the lesion were free from tumor cells and the patient has not reported recurrence till date.

\section{Compliance with Ethical Standards}

Conflict of interest None. 
Ethical Approval This article does not contain any studies with human participants or animals performed by any of the authors.

Informed Consent Informed consent was obtained from all individual participants included in the study.

\section{References}

1. Chummun S, McLean NR, Kelly CG (2001) Adenoid cystic carcinoma of the head and neck. Br J Plast Surg 54(6):476-480

2. Bradley PJ (2004) Adenoid cystic carcinoma of the head and neck: a review. Curr Opin Otolaryngol Head Neck Surg 12(2):127-132

3. Tincani AJ, Negro AD, Costa Araujo PP, Akashi HK, Martins AS, Altemani AS, Barreto G (2006) Management of salivary gland adenoid cystic carcinoma: institutional experience of a case series. Sao Paulo Med J 124(1):26-30

4. Liu WS, Tang PZ, Qi YF, Gao L, Li ZJ (2004) Diagnosis, treatment and prognostic factors of adenoid cystic carcinoma of the palate. Zhonghua Zhong Liu Za Zhi 26:485-489 (Journal in Chinese)

5. Jaso J, Malhotra R (2011) Adenoid cystic carcinoma. Arch Pathol Lab Med 135:511-515
6. Triantafillidou K, Dimitrakopoulos J, Iordanidis F, Koufogiannis D (2006) Management of adenoid cystic carcinoma of minor salivary glands. J Oral Maxillofac Surg 64:1114-1120

7. Li Q, Xu T, Gao J, Ye W, Gu M, Hu W, Wang F, Cai X (2011) Surgery alone provides long-term survival rates comparable to those of surgery plus postoperative radiotherapy for patients with adenoid cystic carcinoma of the palate. Oral Oncol 47:170-173

8. Singh SG, Jain J, Pathak S, Singh KT (2010) Adenoid cystic carcinoma of buccal mucosa. J. Maxillofac Oral Surg 9(3):273-276

9. Gondivkar SM, Gadbail AR, Chole R, Parikh RV (2011) Adenoid cystic carcinoma: a rare clinical entity and literature review. Oral Oncol 47:31-36

10. Neville BW, Damm DD, Allen CM, Bouquot JF (2002) Salivary gland pathology. In: Neville BW, Damm DD, Allen CM, Bouquot JF (eds) Oral and maxillofacial pathology. W.B. Saunders, Philadelphia, pp 389-496

11. Perzin KH, Gullane P, Clairmont AC (1978) Adenoid cystic carcinomas arising in salivary glands: a correlation of histologic features and clinical course. Cancer 42:265-282

12. Szanto PA, Luna MA, Tortoledo ME, White RA (1984) Histologic grading of adenoid cystic carcinoma of the salivary glands. Cancer 54:1062-1069 